\title{
Geolocalizar a los trabajadores no es invasión de su intimidad si el dispositivo utilizado para ello es propiedad de la empresa.
}

\author{
Pilar Rivas Vallejo \\ Catedrática de Derecho del Trabajo y de la Seguridad Social. Universidad de \\ Barcelona
}

Resumen: Geolocalizar a los trabajadores de reparto de comida a domicilio a través de sus propios teléfonos móviles invade el derecho a la privacidad de sus datos, pese a obedecer a un "fin legítimo" relacionado con la tendencia comercial de la competencia, pero lo debería ser, asimismo, aun cuando no se utilizaran tales dispositivos o estos estuvieran a cargo de la empresa.

Palabras clave: Geolocalización. IA. Derecho a la intimidad. Control digital.

Abstract: Geolocating food delivery workers through their own mobile phones invades the right to privacy of their data, despite obeying a "legitimate purpose" related to the commercial trend in food delivery market, but same conclusion should be taken likewise even if such devices were not used or were in charge of the company.

Keywords: Geolocation. Al. Right to privacy. Digital control.

\section{Introducción}

La sentencia analizada confirma la nulidad del proyecto de geolocalización de la empresa Telepizza sobre sus trabajadores de reparto, por basarse esta en el uso de una aplicación instalada en sus propios dispositivos inteligentes, puestos al servicio de la empresa, al comprometerse los datos personales de los trabajadores adheridos a la terminal móvil.

II. Identificación de la resolución judicial comentada

Tipo de resolución judicial: sentencia.

Órgano judicial: Tribunal Supremo, Sala Cuarta.

Número de resolución judicial y fecha: sentencia núm. 163/2021, de 8 de febrero.

Tipo y número recurso o procedimiento: recurso de casación núm. 84/2019.

ECLI:ES:TS:2021:518.

Ponente: Excma. Sra. Dña. María Luz García Paredes.

Votos Particulares: carece. 


\section{Problema suscitado. Hechos y antecedentes}

\section{La nulidad del proyecto de 2018}

Los trabajadores de la empresa Telepizza, S.A.U., a través de sus representantes sindicales, plantean conflicto colectivo con la finalidad de anular el "proyecto tracker" (vigente desde 2018), un sistema de seguimiento de los pedidos de comida a domicilio destinado a mejorar el servicio a los clientes y consistente en geolocalizar los pedidos a través de la propia geolocalización de los repartidores mediante una app que deben instalar en su dispositivo inteligente, exigiendo la empresa a estos que aporten la terminal y la mantengan en uso como herramienta de trabajo y disponiendo consecuencias disciplinarias del incumplimiento de tal deber, en concreto la extinción del contrato. Por ello plantean como pretensión subsidiaria que se aporte por la empresa el dispositivo o se compense adecuadamente, si bien los sindicatos reclamantes CCOO y UGT formulan petita diferentes en algunos extremos. La pretensión principal de nulidad fue estimada por la Sala Social de la Audiencia Nacional, y contra ella recurre la empresa.

\section{Los hechos}

La empresa demandada, Telepizza, SAU, implantó de forma totalmente unilateral en mayo de 2018 el denominado "Proyecto Tracker", consistente en imponer a los trabajadores de reparto (repartidores) de nuevo ingreso en la empresa (a partir del 1 de junio de 2018) la aportación de su propio dispositivo de telefonía inteligente para la realización de su trabajo con el fin de garantizar su geolocalización por aquella durante el tiempo de trabajo mediante una aplicación (app) a disposición de los clientes usuarios de tal app o que realicen los pedidos por internet (a fin de garantizarles el conocimiento en tiempo real del estado de su pedido y mejorar los tiempos de entrega). El sistema de seguimiento implica la obligación de instalar la aplicación y de activarla durante toda la jornada de trabajo, así como de permitir el acceso a la geolocalización y a la cámara del teléfono, sin la cual no funciona el sistema de escañero por código QR que permite identificar a los empleados en la misma para el seguimiento de los pedidos, si bien en la práctica el sistema se ha sustituido por un código numérico aleatorio de seis dígitos, que se calcula en el momento anterior a la asignación de los pedidos, y que no se asocia al empleado de forma permanente, sino que tiene una validez de ocho horas, y tampoco se accede a ningún otro dato personal del teléfono (hecho probado decimoquinto). Los únicos datos captados son la posición del dispositivo, borrándose al día siguiente todos los datos de las coordenadas de la jornada anterior, según indica el informe pericial, de forma que solo los encargados de reparto tienen conocimiento de la asignación, mientras que los datos de la plataforma informática solo están en manos de los técnicos de la empresa que la gestiona (Vector).

El proyecto comporta la inclusión de tal cláusula en su contrato de trabajo de manera obligatoria $(\mathrm{y}$, asimismo, que la negativa reiterada o imposibilidad sobrevenida de aportación de esta herramienta por parte de los trabajadores, o de la aplicación informática, sería causa suficiente para la extinción del contrato de trabajo al amparo del art. 49.1.b) ET transcurrido un plazo de diez días sin reparación o sustitución) y compensación económica por "desgaste de útiles o herramientas".

La representación sindical del comité intercentros se opuso al proyecto en la reunión mantenida con la empresa en julio de 2018, denunciando los hechos a la Inspección de Trabajo (que notificó a la empresa un requerimiento incumplido por esta, por entender que se establecian condiciones de trabajo inferiores a las establecidas legalmente o por convenio colectivo, en contra de lo dispuesto en el art. 3.1.c) ET, concretamente a la LOPD y a los arts. 58, 64.5 y 20.2 ET y 61.13 del convenio aplicable) e interponiendo finalmente el conflicto colectivo por parte de los sindicatos CCOO y UGT, con el fin de declararlo nulo por no haber sido objeto de consulta con la representación legal del personal y por exigir la puesta a disposición de la empresa del dispositivo de telefonía móvil privado (y con acceso a internet) de los trabajadores, no prevista en el convenio aplicable (Convenio Colectivo estatal de elaboradores de 
productos cocinados para su venta a domicilio de 2016). Con posterioridad a este, por parte de la dirección de la empresa se propuso (reunión de septiembre de 2018) incrementar las compensaciones a los trabajadores por "desgaste de útiles o herramientas" y modificar el régimen disciplinario (ampliando hasta catorce días el plazo para reparación o sustitución del terminal), compensaciones que se ofrecieron en reunión posterior, de noviembre de 2018 (hasta 5,50 euros mensuales por la terminal y hasta 2,95 euros por el tráfico de datos).

La sentencia de la que trae causa la que se analiza, dictada por la Sala Social de la Audiencia Nacional en fecha de 6 de febrero de 2019, núm. 13/2019, núm. autos 318/2018 (ECLI:ES:AN:2019:136), declara la nulidad del proyecto sin entrar en las pretensiones subsidiarias o complementarias (según cada demanda). Para ello, califica la geolocalización como tratamiento de datos personales, y concluye que el uso para tal fin de un dispositivo de propiedad de los trabajadores, no ya la geolocalización en sí, vulnera el derecho a la intimidad de los trabajadores, por no superar el juicio de proporcionalidad, ya que esta podría realizarse con mecanismos de ubicación de los vehículos de reparto que no impliquen cesión de datos personales, y por no respetar los deberes informativos que en materia de gestión de datos personales impone la legislación de protección de datos, tanto la que debe proporcionarse a los trabajadores como a su representación legal. Del mismo modo, declara la nulidad del proyecto Tracker por constituir manifiesto abuso de derecho empresarial y quiebra de la nota de ajenidad en el contrato de trabajo, amén de la insuficiencia de la compensación por la aportación de la terminal, calculado sobre precios de baja gama, obligando a los trabajadores asimismo a la contratación de datos. Finalmente, considera que el régimen disciplinario previsto como cláusula de resolución del contrato constituye creación de un régimen de faltas y sanciones que excede los límites del art. 58 ET y que implica modificar el régimen del despido disciplinario, al prescindir de la culpabilidad.

\section{Posición de las partes}

En la fase de instancia, los sindicatos demandantes CCOO y UGT solicitan la nulidad del proyecto Tracker, y, subsidiariamente en caso de UGT, que el dispositivo en cuestión se ponga a disposición por parte de la empresa y no al revés. Asimismo, en segundo grado de subsidiariedad (de forma subsidiaria a la pretensión subsidiaria) pero igualmente acumulativa en el caso de $\mathrm{CCOO}$, solicitan la nulidad de la imposición unilateral por la empresa de la compensación económica por el uso de la terminal propiedad de cada trabajador, alegando que tal compensación debería consistir en la cuantía de la amortización efectiva de la misma de su coste de adquisición por el trabajador y los gastos reales y efectivos de la contratación de transmisión de datos vía internet. Finalmente, CCOO añade la pretensión de nulidad de la medida disciplinaria prevista en el proyecto e incorporada a los contratos de trabajo como causa de extinción de estos y, en segundo lugar, la falta de negociación sobre el proyecto impugnado.

Para sustentar tal petitum, argumentan la ausencia de fundamento acerca de tal obligación (ni siquiera en el convenio colectivo, que sí se refiere a la aportación del uso de vehículo), la introducción de una causa de extinción del contrato abusiva y arbitraria, la existencia de precedentes en la anterior sentencia de 28 de enero de 2014 y la del Tribunal Supremo de 21 de septiembre de 2015 contrarios a la posibilidad de exigir a los trabajadores que proporcionen su teléfono móvil y cuenta de correo electrónico, la existencia de una intromisión en la privacidad de los trabajadores constitutiva de vulneración del art. 18, apdos. 1 y 4, CE, y la insuficiencia de la compensación económica por el sacrificio asumido por los trabajadores, que comporta un enriquecimiento injusto de la empresa (UGT). A lo que CCOO añade que incumple los requisitos de información y consulta del art. 64.5 ET y solicita la nulidad de la cláusula litigiosa incorporada a los contratos, así como de las medidas disciplinarias previstas en el proyecto Tracker.

Por su parte, la empresa Telepizza emplea cinco argumentos, conectados cada uno de ellos con elementos diferentes: la compensación de la aportación del teléfono 
inteligente, la consulta con la representación legal de los trabajadores, la mejora del servicio ofrecido, el derecho a la intimidad y la causa de extinción del contrato:

- Con la incorporación de la cláusula a los contratos de trabajo y su compensación: en este caso, sostiene que la compensación por la aportación del teléfono particular consta reflejada en el contrato de trabajo desde el 20 de diciembre de 2018, y que tal extremo forma parte de la propia oferta de trabajo que los trabajadores aceptan (art. $26.3 \mathrm{ET}$ ), así como que su cuantía es suficiente, por calcularse sobre el precio de 110 euros de un teléfono con geolocalización y vida estimada de tres años (es decir, a razón de 3 euros mensuales más el coste del tráfico de datos, compensado con 64 céntimos al mes, el doble del que se calcula genera el derivado de su uso a tal fin).

- Con la negociación del proyecto: sostiene sí existió negociación sobre ciertos aspectos del proyecto, aunque no sobre el proyecto en sí, que, por otra parte, se viene admitiendo en el marco del art. 20.1 ET sin necesidad de previa negociación colectiva, bajo la condición del respeto de los derechos derivados del tratamiento de los datos personales.

- Con la necesidad empresarial de contar con recursos que permitan mejorar el servicio y aumentar su competitividad: sostiene como argumento en favor de su implantación la aplicación de los mismos sistemas por las empresas competidoras, principalmente Dominos Pizza, y que la finalidad del sistema de geolocalización no reside en las facultades de control empresarial, sino en la mejora del servicio ofrecido al mercado, al permitir a los clientes conocer en todo momento el estado de su pedido, de suerte que no se geolocaliza a los trabajadores, sino a los pedidos, por lo que no se activa la aplicación hasta que el pedido sale del centro de trabajo para su reparto.

- Con el derecho a la intimidad de los trabajadores: se argumenta que la aportación de herramientas y útiles de trabajo, y, en particular, de teléfonos inteligencias propios de la vida personal de los trabajadores, está normalizada en el ámbito laboral, y admitida por la jurisprudencia del Tribunal Supremo, y, por otra parte, que el sistema litigioso no accede a datos personales ni la aplicación se habilita para ello, pues se limita a permitir que los clientes geolocalicen su pedido.

- Con la incorporación de una causa extintiva a los contratos, alega que se trata de causa válida al amparo del art. 49 b) ET.

En la fase de recurso, la empresa ampara su recurso en tres motivos o argumentos: a) la vulneración del del art. 64.5 y 6 ET y la jurisprudencia recogida en la STS de 2 de noviembre de 1999 (rec. 1387/1999), por considerar que ni el ET ni el convenio colectivo aplicable incluyen entre la información que debe facilitarse a la representación legal de los trabajadores la relativa a cuestiones como la debatida, por no comportar un nuevo sistema organizativo; b) la necesidad de la implantación del sistema de geolocalización, que permite superar el juicio de proporcionalidad, no alterado por la titularidad de los dispositivos móviles que deben facilitar los trabajadores, y respetado el derecho de estos a ser informados de ello, puesto que se incorpora al clausulado del contrato de trabajo; c) la ajenidad no queda desvirtuada por la aportación de elementos, útiles o herramientas de trabajo por parte de los trabajadores, prevista incluso en el art. 44 del convenio colectivo aplicable para las motocicletas (prevista en el art. 26.2 ET y admitida por la STS de 26 de febrero de 1986), por lo que la sentencia impugnada contraviene el art. $26.3 \mathrm{ET}$, sin que exista criterio legal o jurisprudencial respecto de la suficiencia de la cuantía de la 
compensación económica por ello, pese a lo cual ha intentado negociar una mejora superior a las cuantías inicialmente ofrecidas.

Por su parte, los demandantes impugnan el recurso, negando la existencia de negociación más allá del proceso de mediación que ellos mismos han abierto (UGT), a lo que CCOO añade que el proyecto fue concebido como un sistema de organización del trabajo por parte de la empresa. En segundo lugar y por lo que respecta al segundo motivo del recurso, oponen el abuso de derecho en que consiste la obligación de aportar el propio dispositivo móvil ya fue objeto de rechazo por la STS de 21 de septiembre de 2015, en relación con la STC 39/2016 (UGT), así como la necesidad del sistema, que no justifica la protección de los datos personales de los trabajadores (obligatorios para la descarga de la aplicación), y la unilateralidad de su implantación, $y$, finalmente, que las cláusulas contractuales impugnadas no respetan el deber de consentimiento informado (CCOO). En tercer y último lugar, se alega por UGT que el material que aportan los trabajadores no es accesorio o marginal, ni tampoco está amparado por norma convencional (que sólo se refiere al vehículo y no a dispositivos inteligentes), y que la cláusula extintiva excede los límites del art. 58 ET. A lo cual CCOO añade que el régimen de compensación económica beneficia a la empresa, amén de imponerse unilateralmente, reiterando los argumentos disciplinario y extintivo ya mencionados.

Finalmente, por parte del Ministerio Fiscal se ataca principalmente por motivos relacionados con la falta de información al comité intercentros en relación con la valoración del impacto sobre los datos de carácter personal, así como con la naturaleza de los datos comprometidos, de carácter personal, y el régimen disciplinario impuesto por la empresa.

\section{Normativa aplicable al caso}

Reglamento 679/2016, de protección de datos de carácter personal (RGPD), arts. 12 y 13.

Convenio Europeo de Derechos Humanos, art. 8.

Constitución española, art. 18. y 90.

Ley orgánica 19/1999, art. 5, y vigente LO 3/2018, de protección de datos, arts. 11

Estatuto de los Trabajadores (ET), arts. 20.1 y 49 b) y 58.

\section{Doctrina básica}

Las cuestiones analizadas en la sentencia se relacionan con tres elementos básicos: a) la quiebra de la ajenidad de la relación como consecuencia de la aportación de herramientas básicas de trabajo; b) la necesidad del sistema de geolocalización para la prestación del servicio; c) el compromiso de los derechos fundamentales; d) la naturaleza organizativa de la medida y los mecanismos para su implantación.

1. La calificación como modificación de sistema organizativo que comporte la información a la representación legal de los trabajadores

El sistema de seguimiento de pedidos por geolocalización se dirige a la satisfacción de los clientes, pero afecta a la organización y control del trabajo, circunstancia que parece incluso admitir la empresa, al informar de la necesidad de informar y formar a los trabajadores acerca de este "nuevo sistema de organización y control de la actividad de reparto". Y, siendo un sistema que, además, impone a los trabajadores el deber de aportar su propio dispositivo móvil y conlleva un posible control de sus datos de carácter personal, con clara repercusión sobre sus condiciones de trabajo (compensaciones económicas), régimen disciplinario y extinción del contrato, cumple las condiciones fijadas en el art. 64.5 ET para considerarlo competencia informativa de la representación legal de los trabajadores. 
Ello por cuanto el art. 64.5 ET establece el derecho de información a la representación legal de los trabajadores (que el art. 67 del convenio aplicable predica del comité intercentros de tratarse de una medida de afectación a más de un centro de trabajo) acerca de todas las decisiones de la empresa que pudieran provocar cambios relevantes en cuanto a la organización del trabajo y a los contratos de trabajo en la empresa, así como a emitir informe con carácter previo a la ejecución de la medida, en particular en la "implantación y revisión de sistemas de organización y control del trabajo, estudios de tiempos, establecimiento de sistemas de primas e incentivos y valoración de puestos de trabajo".

Y tal obligación se incumple si se facilita una información parcial o genérica, sin concretar los aspectos sustantivos del nuevo sistema, como los referentes a los términos de aportación del dispositivo móvil o los hipotéticos datos personales comprometidos, sin que ello suponga, en los términos aludidos en la STS de 2 de noviembre de 1999 (rec. 1387/1999), interferencia en la gestión de la empresa. Y en mayor medida si se hace a posteriori, una vez implantado efectivamente el cambio.

\section{Ajenidad y obligación de aportar el propio dispositivo móvil}

Si bien la aportación de útiles o herramientas de trabajo no ha obstaculizado la calificación como laboral de una prestación de servicios en la jurisprudencia del Tribunal Supremo, esta reseña en resoluciones anteriores, citadas por la que ahora se analiza, y concretamente la STS de 21 de septiembre de 2015 (rec. 259/2014), que tal doctrina se basa en la naturaleza de la aportación de tales medios, de suerte que esta debe comportar una mera aportación, mas no todo el conjunto de responsabilidades en torno a su uso y mantenimiento, como se exige en este caso, en el que además el incumplimiento de tales obligaciones, que se hacen recaer sobre los trabajadores y no sobre la empresa, acarrea consecuencias graves para la propia continuidad del contrato de trabajo. Es este elemento el que pone en duda la ajenidad de la relación, pues no se desvirtúa la ajenidad cuando tales elementos no son elementos esenciales para la configuración y definición del contrato (STS de 25/09/2020, rcud 4746/2019) (FD $3^{\circ}$ de la sentencia estudiada). La jurisprudencia ha ido adaptándose a la evolución tecnológica, aceptando los sistemas de control digital del trabajo, por lo que debe aceptarse la incorporación de tecnología a la vida del contrato, tanto por parte de la empresa como instrumento de control como por parte de los trabajadores, pues algunas tecnologías se han integrado en la vida personal y se han convertido en elementos inescindibles de la propia persona, como ha ocurrido con los dispositivos móviles, y de ahí que también se haya normalizado su uso en el ámbito del trabajo y durante la jornada de trabajo. Lo que ocurre en el caso analizado es que tal normalización ha dado paso a una cultura de empresa que asocia los dispositivos móviles con otros elementos indisociables de la persona como la indumentaria y adopta una postura pragmática por la cual, asumiendo que todos los trabajadores cuentan con tales dispositivos, resulta más lógico adaptar el sistema de control a estos, en lugar de proporcionar sistemas propios a disposición de aquellos, y anuda exigencias adicionales aun más abusivas a esta opción, como es la obligación de contratar datos móviles, lo cual no se encuentra tan normalizado como la propia titularidad de un dispositivo inteligente.

En definitiva, la exigencia de aportación del teléfono móvil "desdibuja la ajenidad e incurre en un abuso empresarial". De suerte que esta distorsión de los términos de la relación laboral es en realidad una forma de conversión del contrato en un arrendamiento de servicios en cuanto al elemento de la ajenidad, mientras permanecen inalterables el resto de los rasgos del contrato de trabajo. Sin embargo, no es esta la intención empresarial, cuya ordenación pragmática únicamente persigue obtener una utilidad de una realidad normalizada, como es la posesión de un dispositivo móvil, como alternativa a la dotación de recursos propios para conseguir el mismo fin, la geolocalización de los pedidos con el objeto de ofrecer un mejor servicio a los clientes. Otra cuestión es que informar a los clientes sobre el recorrido del vehículo que les ofrece la entrega sea más útil que proporcionar una información fiable sobre la hora aproximada de la entrega. 
3. La necesidad y normalización de la geolocalización para la mejora de la competitividad: impacto sobre los derechos fundamentales

Los datos relativos al posicionamiento geográfico que proporciona el uso de un sistema de geolocalización constituyen datos de carácter personal, afirma la sentencia analizada, sobre la premisa de que esta calificación parece ser admitida por ambas partes, lo que implica asumir que tales datos se encuentran amparados por el art. 18 de la Constitución, en sus apartados 1 y 4. Basta con examinar el art. 4.1 del Reglamento 2016/679 para constatar que se atribuye tal naturaleza a los datos de localización y que su captación y registro constituye "tratamiento" a tenor del art. 4.2 del mismo reglamento.

Por tanto, su captación y recogida está sujeta al previo consentimiento del interesado y al suministro de la información necesaria para que este conozca el uso y destino de los mismos o "poder de disposición sobre los datos" (STS de 7 de febrero de 2018, rec. 78/2017, y SSTC 254/1993 y 292/2000).

A partir de ahí, resulta necesario que tal captación y tratamiento responda al juicio de idoneidad y proporcionalidad. Este juicio no debe proyectarse sobre la necesidad del uso de sistemas de geolocalización, admitido que el uso extensivo de tales mecanismos en la sociedad de servicios actual ha dotado a su introducción de necesaria, en el marco de la competitividad entre las empresas, sino sobre el modo en el que se diseña y aplica la medida (test de idoneidad). $Y$ en este segundo ámbito es donde falla el test referido (de proporcionalidad), puesto que lo que no es imprescindible es que la geolocalización (admitida) se incorpore a dispositivos móviles propiedad de los propios trabajadores, con clara invasión de su intimidad, que no deriva de la posibilidad de geolocalizarlos, incluso los clientes o usuarios (que no se cuestiona), sino del acceso por parte de la empresa a los datos personales provenientes de la terminal inteligente que los trabajadores deben poner a disposición de aquella. Test que no puede resultar superado por la contraprestación económica prevista, especialmente por su insuficiencia y falta de acuerdo sobre ella. Y no lo supera porque resulta factible aplicar otros medios "menos invasivos" (v.g. incorporar el mecanismo de geolocalización a los vehículos o a pulseras inteligentes), acordes con el art. 20 bis ET.

Este sistema, por tanto, resulta constitutivo de abuso de derecho (art. 7.2 Cc), porque, como ya mantuvo la STS de 21 de septiembre de 2015, rec 259/2014, resulta abusiva la cláusula contractual que exige a los trabajadores facilitar datos personales para facilitar la comunicación que no son necesarios para el cumplimiento del contrato, lo que excluye la prestación de un consentimiento libre y voluntario, por derivarse tal obligación del propio clausulado.

\section{Parte dispositiva}

La Sentencia desestima el recurso y confirma la sentencia de instancia, por tanto, mantiene la nulidad del proyecto Tracker o de geolocalización de los trabajadores a través de su propio dispositivo inteligencia, en las condiciones abusivas en las que se impuso unilateralmente por la empresa.

\section{Pasajes decisivos}

a) Sobre la admisión del sistema de geolocalización: “...dicha resolución no cuestiona que la geolocalización por la que se va a tener un seguimiento del pedido que debe entregar el repartidor al cliente no sea un método adecuado o idóneo a tal finalidad -se dice por la Sala de instancia que la medida obedece a fines constitucionalmente legítimos en el desarrollo a la libre empresa como son el control del empleado en el desempeño de su puesto de trabajo y la oferta de un mejor servicios al cliente, (f.d. sexto)- sino que la configuración dada al mismo por la demandada no es conforme a derecho. Por tanto, no está negando que sea una medida legitima para determinados fines o que con ella no se vaya a evitar un déficit comercial competitivo, en un momento en el que, ciertamente, ese sistema de prestar el servicio al cliente se extiende en este y otros sectores sino, simplemente, que el que 
ella ha implantado no supera los criterios constitucionales ni legales, cuando existen otras formas de ejecutar ese sistema que no sea ese" (FD $2^{\circ}$ ).

b) Sobre el test de proporcionalidad y la existencia de otras alternativas no invasivas (el uso del mismo sistema pero incorporado a medios que no comprometan datos personales): "...el test de idoneidad, desde la perspectiva de los derechos fundamentales, que es a lo que se refiere la sentencia recurrida, no es el que ha impedido superar el juicio de proporcionalidad sino el test de necesidad, en el sentido de que el medio o instrumento al que ha acudido la empresa para obtener aquel objetivo no es adecuado por existir otros medios menos invasivos y, en este extremo, la recurrente no ha desvirtuado lo que al respecto señala la Sala de instancia, al indicar que existen otros instrumentos que, siendo adecuados para cubrir la finalidad que persigue la empresa, resultan ser no invasivos de aquellos derechos constitucionalmente protegidos" (FD $2^{\circ}$ ).

c) Sobre la ajenidad de la prestación laboral como consecuencia de la digitalización del trabajo: "debemos señalar que recientemente esta Sala ha recordado la evolución que están experimentando las relaciones contractuales en las que existen prestaciones de servicios a consecuencia de las innovaciones tecnológicas y, concretamente, en aspectos que afectan a sistemas de control digitalizados de los servicios que se prestan y que ello obliga a tener que adaptar los conceptos que caracterizan, en este caso, las relaciones laborales, recordando, ciertamente, que la aportación de elementos o herramientas para la prestación de servicios no desvirtúa la ajenidad cuando aquellos no son elementos esenciales para la configuración y definición del contrato (STS de 25/09/2020, rcud 4746/2019)" (FD 3).

"...cuando hace mención del concepto ajenidad en los medios lo realiza no solo desde la mera puesta a disposición por el trabajador del móvil sino atendiendo también a lo que se añade respecto del uso de dicho elemento, al imponerse en el proyecto efectos sobre el contrato de trabajo ante cualquier alteración que el dispositivo sufra y no permita la conexión, trasladando al trabajador la responsabilidad hasta el punto de que puede llegar a suspender la relación laboral con pérdida de salario o, incluso, extinguirla. Es este el marco en el que la aportación del móvil, según la sentencia recurrida, desdibuja la ajenidad e incurre en un abuso empresarial" (FD $3^{\circ}$ ).

d) Sobre el abuso de derecho (art. 7.2 Cc): "ha sido objeto de análisis en supuestos que se asemejan al presente, en tanto que afectan a datos de carácter personal, como ya recogen los demandantes, con cita de la STS de 21 de septiembre de 2015, rc 259/2014, que califica de abusiva una cláusula de contrato en la que se exige que el trabajador facilite datos a efectos de mantener contacto y remisión de comunicaciones que afectan al contrato, así como que cualquier cambio deberá ser notificado a la empresa de forma fehaciente e inmediata. En ese caso se dijo que no se puede entender que exista consentimiento libre y voluntario del trabajador al exigir el clausulado del contrato la aportación por parte del trabajador de aquellos datos teléfono o email-, no necesarios ni imprescindibles para el cumplimiento del contrato" $\left(\right.$ FD $\left.3^{\circ}\right)$.

\section{Comentario}

\section{El uso de inteligencia artificial para la gestión de las relaciones de trabajo}

En la actualidad, las formas más domésticas y comerciales de la inteligencia artificial, lejos de los objetivos a largo plazo de la inteligencia computacional o cognitiva, se han introducido en la gestión cotidiana de las empresas, y, por tanto, de las relaciones de trabajo. Las distintas aplicaciones de gestión, llamadas "inteligencia de negocios", que ofrecen las empresas del sector permiten mejorar la eficiencia y rapidez de los distintos procesos y tareas que comporta el desarrollo de la actividad empresarial, y, entre ellas, se encuentran aquellas destinadas al seguimiento de los trabajadores a través de distintos métodos.

No cabe duda de que el control de los trabajadores a través de dispositivos inteligentes puede encontrarse en esa línea de gestión digital de la empresa, y, por 
tanto, que el empleo de este tipo de dispositivos puede manejar inteligencia artificial a pequeña escala, usos comerciales encuadrables dentro del concepto genérico de "tecnología". Por el contrario, los sistemas de geolocalización a través de posicionamiento global no forman parte de esa tipología de herramientas, por basarse en un sistema "más clásico" de posicionamiento de satélites (aunque los sistemas GPR utilizan igualmente sofware, pues se definen por ser un "conjunto de hardware y software diseñados para determinar localizaciones precisas sobre la superficie terrestre utilizando señales procedentes de una serie de satélites seleccionados", "midiendo las distancias desde múltiples satélites que están orbitando alrededor de la Tierra para registrar las coordenadas x, y, z de la posición de un receptor GPS").

No obstante, si este sistema se incorpora a un dispositivo inteligente, puede no solo proporcionar un posicionamiento a su usuario, sino permitir su detección por terceros, además de cruzar ambos tipos de datos, y direccionarlos para su seguimiento por tales terceros. De suerte que son los datos que incorpora el teléfono inteligente los que resultan transmitidos hasta quien tenga acceso a su posicionamiento, vinculando, pues, datos y geolocalización.

Según argumenta la sentencia comentada, siendo este tipo de utilidades común y extendida en la realidad empresarial actual, la vieja sentencia de la sabiduría popular "no podemos poner puertas al campo" avala la admisión del seguimiento digital de los trabajadores, sin apenas cuestionarlo, dada la normalización de su uso en la actual sociedad digital. Y así sucede en la resolución analizada, donde lo que se cuestiona no es el modo de control en sí, al considerarse legítimo el seguimiento por geolocalización cuando se trata del reparto de comida a domicilio, sino a que, además, se realice con invasión de la intimidad y el derecho a la privacidad de los datos personales de los trabajadores. De forma que tal derecho es el que opera como límite absoluto a la disposición de sistemas digitales de control de los trabajadores. Esta tesis, vinculada a la protección de la privacidad de los datos personales, avalaría la improcedencia del empleo de otro tipo de mecanismos de seguimiento durante la jornada de trabajo que sirvieran para dar cuenta de pausas de los trabajadores por necesidades personales (v.g. fisiológicas) y de la comunicación de sus motivos a los empleadores, pues ello sería admitir que las empresas pueden tener acceso a datos relacionados con datos biológicos, perfectamente conectables con otros datos de mayor relevancia, como es la detección de enfermedades, mediante sistema de minería de datos y algoritmos predictivos. En definitiva, la prevención y límite al uso de estos mecanismos se encuentra, en la doctrina analizada, en el uso de los datos personales, en el marco del art. 22 del Reglamento europeo de protección de datos personales 2016/679.

Ahora bien, no se cuestiona, como se ha indicado, que este control digital se lleve a cabo por medios dispuestos por la empresa, como reza la sentencia comentada, si ello deriva en la prestación de un mejor servicio al cliente, lo que convierte su uso en imprescindible, a tenor de su normalización en el mercado de la competencia al que sirve. Se razona al respecto que esta tendencia, sobre la que se tratará en el apartado siguiente, justifica el seguimiento personal de los trabajadores a través de sistemas de geolocalización. El sistema en cuestión se orienta, al parecer, exclusivamente al seguimiento por parte de los clientes. Ahora bien, en tanto comporta tal control, implica también que se abre la puerta al control directo de la empresa (si los clientes pueden, es obvio que también lo hace la empresa), a efectos de reforzar el cálculo de tiempos $\mathrm{y}$, en definitiva, de rendimiento de los trabajadores, con las consecuencias que ello comporta para su propia evaluación de la diligencia debida en la ejecución del contrato de trabajo. Con ello, se incorpora asimismo la evaluación por terceros, los usuarios o clientes, convertidos en auxiliares de la gestión de personal de este tipo de empresas que admiten las evaluaciones externas, en cuanto los clientes no están evaluando únicamente el producto que ofrece la empresa, sino la ejecución del servicio por los propios trabajadores. ¿Realmente es necesario "normalizar" esta nueva categoría de evaluadores externos del trabajo que desempeña este tipo de trabajadores? 
Por otra parte, cabe cuestionar si la mejora del servicio debe implicar necesariamente el derecho de los clientes a invadir la privacidad de los trabajadores, aunque no se realice mediante el uso de sus propios dispositivos sino de otros titularidad de la empresa, para conocer su ubicación y otros datos (téngase en cuenta que algunas empresas incluso comparten el nombre de pila de los repartidores), si se admite que conocer la ubicación de una persona resulta realmente invasivo de su privacidad. O si, por el contrario, la misma calidad del servicio, en el marco de las tendencias actuales del mercado, puede ofrecerse por mecanismos alternativos, como el suministro a los clientes sobre la hora estimada de la entrega, que suple al sistema de seguimiento personalizado de los repartidores de comida. Precisamente este es el sistema alternativo que siguen otras plataformas de reparto de comida a domicilio, v.g. Just-eat, sin que por ello se resienta ni el servicio ni la competitividad de la empresa. Evidentemente estos razonamientos no se dirigen al contenido de la sentencia, al no corresponder tal función a los tribunales, pero sirven para reflexionar sobre el alcance y necesidad de tales mecanismos de control.

\section{La tendencia del mercado como argumento para la invasión del derecho a la} intimidad

La empresa demandada sostiene que el sistema en conflicto es utilizado de forma generalizada en el sector de la comida a domicilio, y que excluir su uso la sitúa en una posición de debilidad en el mercado, lo que justifica su implantación para garantizar una oferta similar a la de la competencia, argumento aceptado por la sentencia analizada.

En efecto, la sentencia no cuestiona que la geolocalización compartida con terceros, los usuarios, sea razonable, porque se adapta a la "tendencia" en el mercado de comida a domicilio, sino solo que deba realizarse colonizando empresarialmente una esfera puramente privada de los trabajadores: sus teléfonos móviles. Se razona que el uso de dispositivos propiedad de los trabajadores no soporta el test de proporcionalidad, porque implica invadir su privacidad a través de la captación de sus datos personales, o de algunos de ellos, los incorporados al teléfono móvil. Y ello se justifica por razones de competitividad, es decir, porque las empresas del sector están utilizando tales sistemas de seguimiento para la mejora de la prestación del servicio a los clientes, porque es tendencia. Ergo se admite que la digitalización de los servicios permita que terceros ajenos a la empresa puedan invadir la privacidad de los trabajadores detectando en todo momento dónde se ubican. ¿Implica esto admitir, como está resultando práctica habitual por parte de las empresas de reparto de productos de supermercado, también la identificación básica de la persona? V.g. Amazon Prime Now informa a los usuarios sobre el nombre de pila de la persona encargada del reparto, simultáneamente al envío de su geolocalización, además de facilitar después el mecanismo para la evaluación de tal servicio, esto es, la calificación del propio trabajador o trabajadora previamente identificado.

$\mathrm{Si}$ el único argumento de rechazo del seguimiento y geolocalización de los trabajadores es la tendencia del mercado, se está admitiendo en realidad que el argumento de la competitividad prime sobre el control en tiempo real o monitorización de los trabajadores, sin cuestionar que tales mecanismos pueden ser fácilmente sustituibles por otros menos invasivos, como es, simplemente, la notificación de la hora de la entrega a los clientes, que no necesitan ocupar su tiempo en monitorizar la ubicación en tiempo real del repartidor de su comida. Habría de cuestionarse, pues, su propia necesidad como parte de la mejora del servicio ofrecida por la empresa aplicando el test de necesidad y proporcionalidad, en el marco de la Carta de los Derechos Fundamentales (arts. 7 y 8 , derecho al respeto a la vida privada y familiar y a la protección de datos de carácter personal) y del Informe anual A/74/493, 11 de octubre de 2019, del relator especial sobre la extrema pobreza y los derechos humanos, sobre el estado de bienestar digital y los derechos humanos, en el que se traslada a la Asamblea General de Naciones Unidas los riesgos del "estado de bienestar digital" para los derechos humanos (Lazcoz y Carrillo, 2020). Cabe traer a colación la sentencia del Tribunal de distrito de La Haya de 5 de febrero de 2020, en el 
asunto SyRI, sistema de indicación de riesgos, en la que tal tribunal aplicaba el mencionado test a un sistema público, concluyendo que el sistema en cuestión no garantizaba la privacidad de acuerdo con lo previsto en el art. 8 del Convenio Europeo de Derechos Humanos (CEDH), por no superar ni el test de necesidad ni el de proporcionalidad.

Pues bien, resulta harto cuestionable que quepa entender que una herramienta de seguimiento personal como la que centra el litigio supere el juicio de necesidad, y, por ende, tampoco el de proporcionalidad. En la sentencia analizada se afirma que, si tal dispositivo se hubiera instalado en el vehículo o en una pulsera, podría superar el juicio de proporcionalidad, admitido que el mecanismo es necesario en el contexto laboral al que se aplica. Sin embargo, examinada la finalidad a la que se dirige, proporcionar seguimiento del pedido a los clientes, ya se ha aludido a que esta finalidad se puede cumplir a través de otras técnicas que no supongan geolocalizar a los trabajadores. Comoquiera que este es el único objetivo aducido por la empresa, y no el propio control de la ejecución del trabajo a través de su monitorización, no puede concluirse que el objetivo sea necesario, pese a ser legítimo, pues puede sustituirse por otras técnicas igualmente competitivas. En segundo lugar, optar por el uso de una pulsera o reloj de pulsera por los trabajadores (F.D. $6^{\circ}$ de la sentencia de instancia) parece destinado a la misma conclusión, pues pretende dar satisfacción a esa supuesta necesidad de ofrecer el seguimiento de los trabajadores por los clientes (sin perjuicio de que habría de analizarse si esos otros medios alternativos no comportan igualmente la transferencia de datos personales). $Y$ en todo caso requeriría que su uso (aun el de una terminal facilitada por la empresa) se limitara al tiempo y lugar de trabajo, desactivándose fuera de estos parámetros, para evitar el seguimiento durante la vida extralaboral (STS766/2020, de 15 de septiembre [rec. 528/2018], sobre seguimiento por GPS instalado en vehículo de empresa reservado para su uso con fines laborales). Su generalización, en todo caso, debería venir acompañada por la esperable respuesta sindical, como la que ha reaccionado en Reino Unido (CBI y TUC) contra la actividad de BioTeq, fabricante de implantes de chips para el marcaje horario de los trabajadores (sistemas desarrollados en Europa por las empresas suecas Epicenter, o Biohax Internacional, o la belga NewFusion).

Por otra parte, a tenor de este argumento, resultaría igualmente "necesario" geolocalizar a otro tipo de trabajadores prestadores de servicios por parte de los clientes a fin de determinar su ubicación y temporización de la ejecución de tales servicios, v.g. en instalación y reformas, entre otros, abriendo la puerta a que por parte de cualquier cliente se pudiera conocer en tiempo real la ubicación de tales trabajadores en todo momento y, además, puntuar a través del sistema de calificación aplicable, el servicio prestado, incluida su temporalización. Un sistema que convierte a los usuarios o clientes en "prosumidores", que contribuyen con su trabajo, no protegido contra los sesgos y las arbitrariedades, a la calificación del trabajo de tales empleados, convirtiéndose así en auxiliares gratuitos de la gestión de los recursos humanos de la empresa. Ello sin mencionar que abrir esta opción a los clientes podría permitir realizar seguimientos personales con fines ilegítimos, incluidos los basados en violencia de género, pues la herramienta pone en manos de cualquier usuario, sabedor del tamaño y composición de la flota de repartidores con la que cuenta cada sede de la empresa, un instrumento de control que puede llegar a ser perverso si se utilizara con fines delictivos y, en cualquier caso, contrario al art. $8 \mathrm{CEDH}$ en relación con el art. 4.1 del RGPD.

En definitiva, permeabilizar las relaciones de trabajo para incorporar a terceros, los clientes, no encaja en las facultades reservadas al empleador en el Estatuto de los Trabajadores. De concebirse como una externalización de la valoración del trabajo y del rendimiento y la diligencia a "prosumidores", constituiría sin duda una modificación sustancial de las condiciones de trabajo que habría de someterse al preceptivo procedimiento de consultas. Por otra parte, como se razona en la sentencia de la que trae causa la resolución comentada, este mecanismo comporta igualmente atribuir efectos disciplinarios ajenos al régimen jurídico previsto en el art. 58 ET. Concretamente porque la calificación de los trabajadores mediante evaluaciones 
externas tiene una clara repercusión sobre la continuidad de su relación de trabajo, más allá de lo que se enjuicia en el pleito analizado (las consecuencias disciplinarias de no mantener actualizado y operativo el terminal inteligente empleado).

\section{Apunte final}

La sociedad digital está conduciendo a un necesario replanteamiento de las relaciones entre la competitividad y la libre empresa y los derechos fundamentales, que permita hacer compatible el imparable avance de la técnica, la digitalización y los sistemas de gestión basados en la inteligencia artificial y el respeto a los derechos de los trabajadores. Ello implica valorar qué injerencias externas a la propia relación laboral deben tolerarse en pro de la competitividad de las empresas, especialmente en el sector de los servicios, y en particular qué relevancia debe concederse a la valoración externa del servicio prestado por la empresa respecto del trabajo ejecutado por los trabajadores. Por tanto, conviene estudiar los mecanismos de neutralización del impacto negativo de tales injerencias, en lugar de conceder a estos sistemas "reputacionales" un papel preeminente en el sistema de cálculo del rendimiento o la valoración de la diligencia debida en el contrato de trabajo. Y, por otra parte, el ya más clásico concepto de la responsabilidad social de las empresas debe integrar el uso ético de los mecanismos digitales de control, priorizando técnicas que, pudiendo basarse igualmente en tecnología, permitan guardar tal necesario equilibrio. En este marco juega un papel crucial la jurisprudencia de la sala cuarta del Tribunal Supremo, además de la negociación colectiva. 